\title{
Plasmalogen Deficiency and Overactive Fatty Acid Elongation Biomarkers in Serum of Breast Cancer Patients Pre- and Post-Surgery-New Insights on Diagnosis, Risk Assessment, and Disease Mechanisms
}

\author{
Satomi Tomida ${ }^{1,2}$, Dayan B. Goodenowe ${ }^{3}$, Teruhide Koyama ${ }^{2, *} \mathbb{C}$, Etsuko Ozaki ${ }^{2}$, Nagato Kuriyama ${ }^{2,4}$, \\ Midori Morita ${ }^{1}{ }^{1}$, , Yasuyo Yamazaki ${ }^{3}$, Koichi Sakaguchi ${ }^{1}$, Ritei Uehara ${ }^{2}$ and Tetsuya Taguchi ${ }^{1}$ (D) \\ 1 Department of Endocrine and Breast Surgery, Kyoto Prefectural University of Medicine, \\ Kyoto 602-8566, Japan; satomida@koto.kpu-m.ac.jp (S.T.); midori@koto.kpu-m.ac.jp (M.M.); \\ ksak@koto.kpu-m.ac.jp (K.S.); ttaguchi@koto.kpu-m.ac.jp (T.T.) \\ 2 Department of Epidemiology for Community Health and Medicine, Kyoto Prefectural University of \\ Medicine, Kyoto 602-8566, Japan; ozaki@koto.kpu-m.ac.jp (E.O.); nkuriyama@s-sph.ac.jp (N.K.); \\ ruehara@koto.kpu-m.ac.jp (R.U.) \\ 3 Prodrome Sciences USA LLC, Temecula, CA 92591, USA; d.goodenowe@prodromesciences.com (D.B.G.); \\ Y.Yamazaki@prodromesciences.com (Y.Y.) \\ 4 Shizuoka Graduate University of Public Health, Shizuoka 420-0881, Japan \\ check for \\ updates \\ * Correspondence: tkoyama@koto.kpu-m.ac.jp; Tel.: +81-75-251-5789; Fax: +81-75-251-5799
}

Citation: Tomida, S.; Goodenowe, D.B.; Koyama, T.; Ozaki, E.;

Kuriyama, N.; Morita, M.; Yamazaki, Y.; Sakaguchi, K.; Uehara, R.; Taguchi, T. Plasmalogen Deficiency and Overactive Fatty Acid Elongation Biomarkers in Serum of Breast Cancer Patients Pre- and Post-Surgery-New Insights on Diagnosis, Risk Assessment, and Disease Mechanisms. Cancers 2021, 13, 4170. https://doi.org/10.3390/

cancers 13164170

Academic Editor: David Wong

Received: 27 May 2021

Accepted: 16 August 2021

Published: 19 August 2021

Publisher's Note: MDPI stays neutral with regard to jurisdictional claims in published maps and institutional affiliations.

Copyright: (c) 2021 by the authors. Licensee MDPI, Basel, Switzerland. This article is an open access article distributed under the terms and conditions of the Creative Commons Attribution (CC BY) license (https:// creativecommons.org/licenses/by/ $4.0 /)$.
Simple Summary: Breast cancer (BC) is the most commonly diagnosed cancer in women. Mammography and ultrasonography are commonly used for BC screening; however, they are associated with problems such as inconvenience, radiation exposure, and dependence on the skill level of operators. To overcome this problem, we performed a comprehensive lipid metabolomic analysis of serum using high-resolution accurate mass spectrometry from two case-control studies that included non-BC, BC subjects pre-surgery and $\mathrm{BC}$ subjects one-month post-surgery to determine if the metabolic signatures of over-active fatty acid elongation and other lipid changes could be detected in BC vs. non-BC subjects. The ratios of the linoleic acid to the oleic acid which were evaluated in multiple lipid pools were lower in pre-surgery BC subjects, however, these ratios increased at post-surgery and were no longer different from non-BC subjects. On the other hand, the ethanolamine plasmalogen levels were lower in pre-surgery BC subjects and were not recovered by surgical removal. These do not appear to be caused by BC tumor activity and may be pre-existent and a possible risk factor for BC. In this study, we have identified several lipid metabolic systems that detect both BC risk and BC activity.

Abstract: The polyunsaturated fatty acid (PUFA) elongase, ELOVL5, is upregulated in breast cancer (BC) vs. adjacent normal tissue. We performed a comprehensive lipid metabolomic analysis of serum using high-resolution accurate mass spectrometry from two case-control studies that included non$\mathrm{BC}, \mathrm{BC}$ subjects pre-surgery, and BC subjects one-month post-surgery to determine if the metabolic signatures of over-active fatty acid elongation and other lipid changes could be detected in $\mathrm{BC}$ vs. nonBC subjects: study 1 ( $n=48$ : non-BC, $n=69$ : pre-surgery $\mathrm{BC}$ ); study 2 (blinded validation: $n=121$ : non-BC, $n=62$ : pre-surgery $\mathrm{BC}, n=31$ : one month post-surgery). The ratio of the ELOVL5 precursor, linoleic acid (18:2) to a non-ELOVL5 precursor, oleic acid (18:1) was evaluated in multiple lipid pools (phosphatidylethanolamine (PtdEtn), phosphatidylcholine (PtdCho), lyso-PtdCho, and free fatty acids). This ratio was lower in pre-surgery BC subjects in all pools in both studies $(p<0.001)$. At one-month post-surgery, the 18:2/18:1 ratios increased vs. pre-surgery and were no longer different from non-BC subjects ( $p>0.05$ expect for lyso-PtdCho). In contrast to the elongation biomarkers, docosahexaenoic acid (22:6n-3) containing ethanolamine plasmalogen (EtnPls) species were observed to be further decreased in BC subjects one-month post-surgery vs. pre-surgery levels $(p<0.001)$. These results are consistent with the hypothesis that ELOVL5 is upregulated in BC tissue, which would result in the selective depletion of 18:2 vs. 18:1 containing lipid species. Surgical removal of the tumor removes the overactive ELOVL5 effect on serum lipids. In contrast, the low EtnPls levels 
do not appear to be caused by BC tumor activity and may be pre-existent and a possible risk factor for BC. These results indicate that it may be possible to screen for both breast cancer risk and breast cancer activity using a simple blood test.

Keywords: breast cancer; fatty acid; ELOVL5; plasmalogen

\section{Introduction}

Breast cancer $(\mathrm{BC})$ has the highest cancer-related morbidity rate among Japanese women [1]. The five-year survival rate is more than $90 \%$ if $B C$ is diagnosed at stage 1 or 2 . Accordingly, early diagnosis results in a good prognosis. It is important to diagnose BC and initiate treatment as early as possible. However, the $\mathrm{BC}$ screening rate among Japanese people is lower than in other countries (approximately 40\%) [2], and many Japanese women may miss the opportunity for early detection of BC. Mammography and ultrasonography are commonly used for $\mathrm{BC}$ screening; however, they are associated with problems such as inconvenience, radiation exposure, and dependence on the skill level of operators. A minimally invasive and convenient serum-based test that can accurately identify BC patients would increase the screening rate and result in detecting $\mathrm{BC}$ at an earlier stage.

Several epidemiological studies have described the role played by dietary factors in determining the risk of $\mathrm{BC}$ [3-5]. It has been speculated that fatty acids are the dietary component associated with the occurrence of BC [6-9]; however, this remains controversial. A meta-analysis of prospective cohort studies found no association between serum fatty acid levels and risk of breast cancer [7]. On the other hand, some experimental studies found a relationship between fatty acid levels and the risk of BC. The very long-chain fatty acid elongase (ELOVL) plays an important role in fatty acid elongation [10]. Mammals have 7 elongases (ELOVL1-7), each with different substrate specificities [10]. ELOVL5 elongates omega- $3(n-3)$ and omega- 6 (n-6) polyunsaturated acids and prefers $C 18-\mathrm{C} 22$. Yamashita et al. reported that the level of linoleic acid (18:2) was lower in BC tissues and suggested that ELOVL5 accelerated elongation [11]. The researchers also reported that the mRNA expressions of ELOVL1, 5, and 6 were significantly higher in BC tissues than in corresponding normal breast tissues [11].

We have previously described the low levels of 28 carbon polyhydroxylated and the polyunsaturated long-chain fatty acid $\left(\mathrm{C}_{28} \mathrm{H}_{46} \mathrm{O}_{4}\right)$, named GTA-446 in colorectal cancer patients, and the low levels of 36 carbon polyhydroxylated and the polyunsaturated longchain fatty acid $\left(\mathrm{C}_{36} \mathrm{H}_{66} \mathrm{O}_{6}\right)$, named PTA-594 in pancreatic cancer patients [12-15]. We also described low levels of plasmalogens in both patients [12-15]. To identify the metabolic signatures of up-regulated fatty acid elongation and other lipid changes in $\mathrm{BC}$ vs. non-BC subjects, we performed a comprehensive lipid metabolomic analysis of serum using highresolution accurate mass spectrometry in two case-control studies in completely different cohorts that included non-BC and BC pre-surgery subjects as well as $B C$ subjects one month after surgery.

\section{Results}

\subsection{Discovery Phase}

Table 1 shows the characteristics of the enrolled BC patients and healthy controls. Comprehensive, non-targeted high-resolution mass spectrometry of the discovery phase (study 1) serum samples resulted in over 2000 unique sample-specific accurate masses. Accurate masses corresponding to specific species of free fatty acids (FFA), phosphatidylethanolamine (PtdEtn), phosphatidylcholine (PtdCho), lyso-PtdCho, and ethanolamine plasmalogens (EtnPls) were observed to have significantly different levels in BC vs. non-BC persons (Table S1). Figure S1 shows 18:2 (linoleic acid)/18:1 (oleic acid) ratios are significantly lower with the $p$-value $<0.0005-0.000001$ between BC patients and healthy controls on the red plots; metabolites of FFA, lyso-PtdCho, PtdEtn, and PtdCho series. Based on this 
analysis, a subset of lipid metabolites of interest was then created using key representative species of each of these metabolite classes for subsequent analyses and validation.

Table 1. Characteristics of study 1 and study 2.

\begin{tabular}{ccccc}
\hline & \multicolumn{2}{c}{ Study 1 } & \multicolumn{2}{c}{ Study 2 } \\
\hline & Breast Cancer & Control & Breast Cancer & Control \\
\hline Total & 69 & 48 & 62 & 121 \\
Age (SD) & $59.84(13.70)$ & $61.58(9.07)$ & $59.59(13.79)$ & $59.26(13.32)$ \\
(Range) & $(34-91)$ & $(41-74)$ & $(34-78)$ & $(39-74)$ \\
Stage 0 $(n)$ & $26.1 \%(18)$ & - & $22.6 \%(14)$ & - \\
Stage 1 $(n)$ & $42.0 \%(29)$ & - & $41.9 \%(26)$ & - \\
Stage 2 $(n)$ & $20.3 \%(14)$ & - & $33.9 \%(21)$ & - \\
Stage 3 $(n)$ & $5.8 \%(4)$ & - & $1.61 \%(1)$ & - \\
Stage 4 $(n)$ & $5.8 \%(4)$ & - & $0 \%(0)$ & - \\
\hline
\end{tabular}

\subsection{Biochemical System Validation}

All study 1 samples along with all study 2 samples, which were sent to the laboratory blinded, were then processed in a manner identical to that of the original discovery phase; however, only the pre-selected lipid metabolites of interest were investigated. The original lipid metabolomic system changes observed in the discovery phase were re-affirmed upon re-analysis, attesting to the reproducibility of the extraction and analysis procedures. After receiving the data from the laboratory, the identities of study 2 samples were revealed, and the results were interpreted with respect to study cohort, disease stage, and pre-surgery vs. post-surgery status.

\subsection{Validation Cohort Analysis}

The effect of BC on the lipid metabolomic system was observed to be similar in both the discovery and validation data sets (Table S2). Figure 1 illustrates the reproducibility of the lower 18:2/18:1 ratio across multiple lipid pools in both study cohorts. To further illustrate and compare the selective depletion of 18:2 species and EtnPls in BC, representative pairs of PtdEtn and ethanolamine plasmalogens (PlsEtn) species were normalized to PtdEtn 18:0/18:1 (Figures 2 and 3). For details about this normalization, see Materials and Methods. The level of PtdEtn 18:0/18:1 was unchanged in BC and control subjects; however, both the levels of PtdEtn 18:0/18:2 and EtnPls 18:0/18:2 species were decreased in BC subjects relative to control (Figure 2). Likewise, the levels of key polyunsaturated arachidonic acid $(20: 4 n-6)$ and docosahexaenoic acid (22:6n-3) were decreased, and this decrease was more severe in the EtnPls lipid pool. Figure 3 illustrates the selective effect of surgical tumor removal on the restoration of 18:2 species in both the PtdEtn and EtnPls pools; however, the levels of polyunsaturated n-6 and n-3 species were either unaffected or further reduced in post-surgery. Figure 4 illustrates the reproducibility of the normalizing effect of surgery on the 18:2/18:1 ratio in multiple lipid pools. In addition to EtnPls species and 18:2 containing phospholipids, lyso-PtdCho species were also observed to be reproducibly decreased in $B C$ vs. non-BC subjects (Figure 5). No effect of the cancer stage was observed for any of the biomarkers investigated. Figure 6 illustrates the lack of effect of the cancer stage on several key biomarkers described above. 


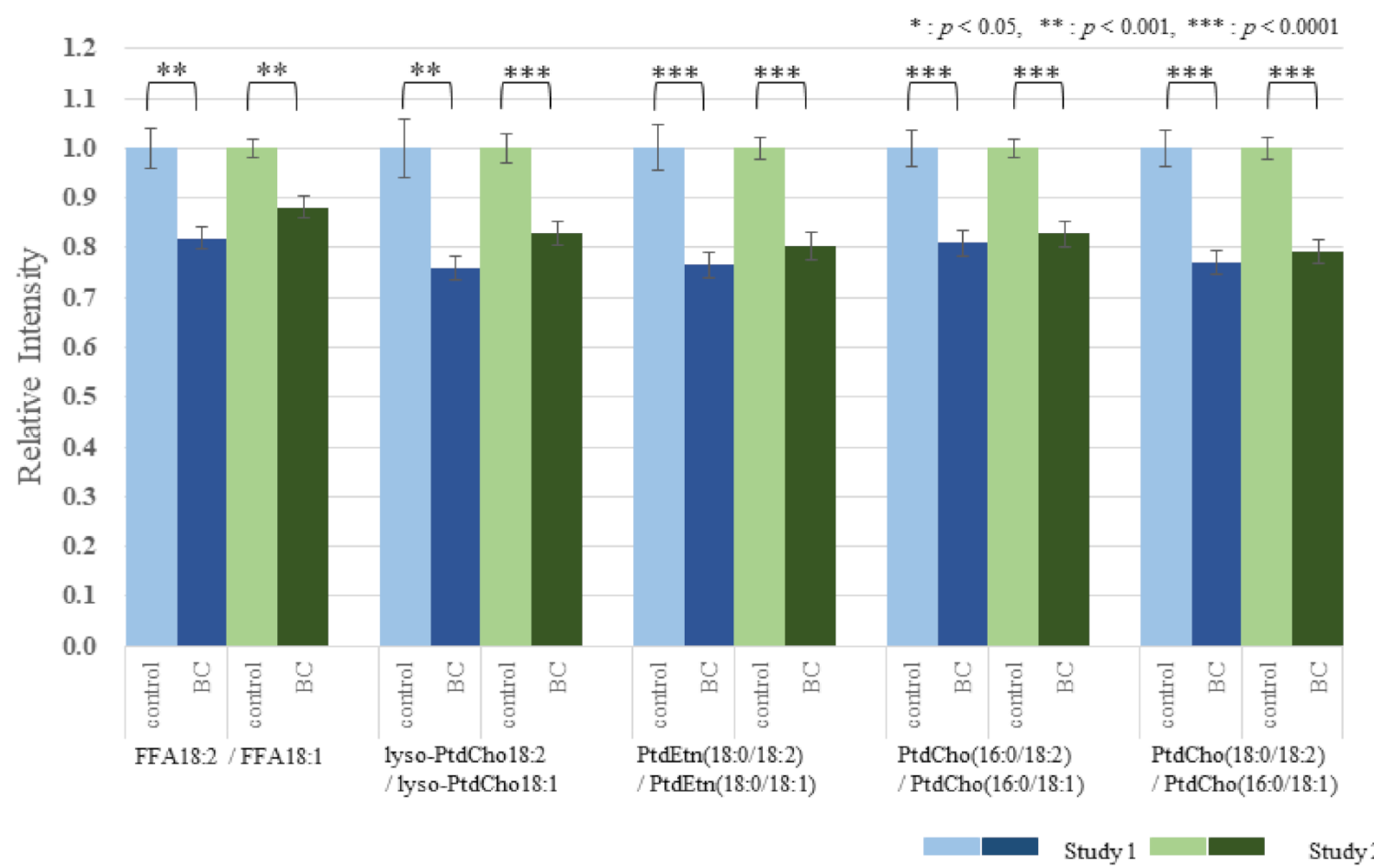

Figure 1. Similar results in both the discovery and validation study, the lower ratio of each species containing 18:2 to each species containing 18:1 (18:2/18:1 ratio) across multiple lipid pools in pre-surgery BC relative to the 18:2/18:1 ratios in control. (BC means are described as relative intensity when control means were 1.00).

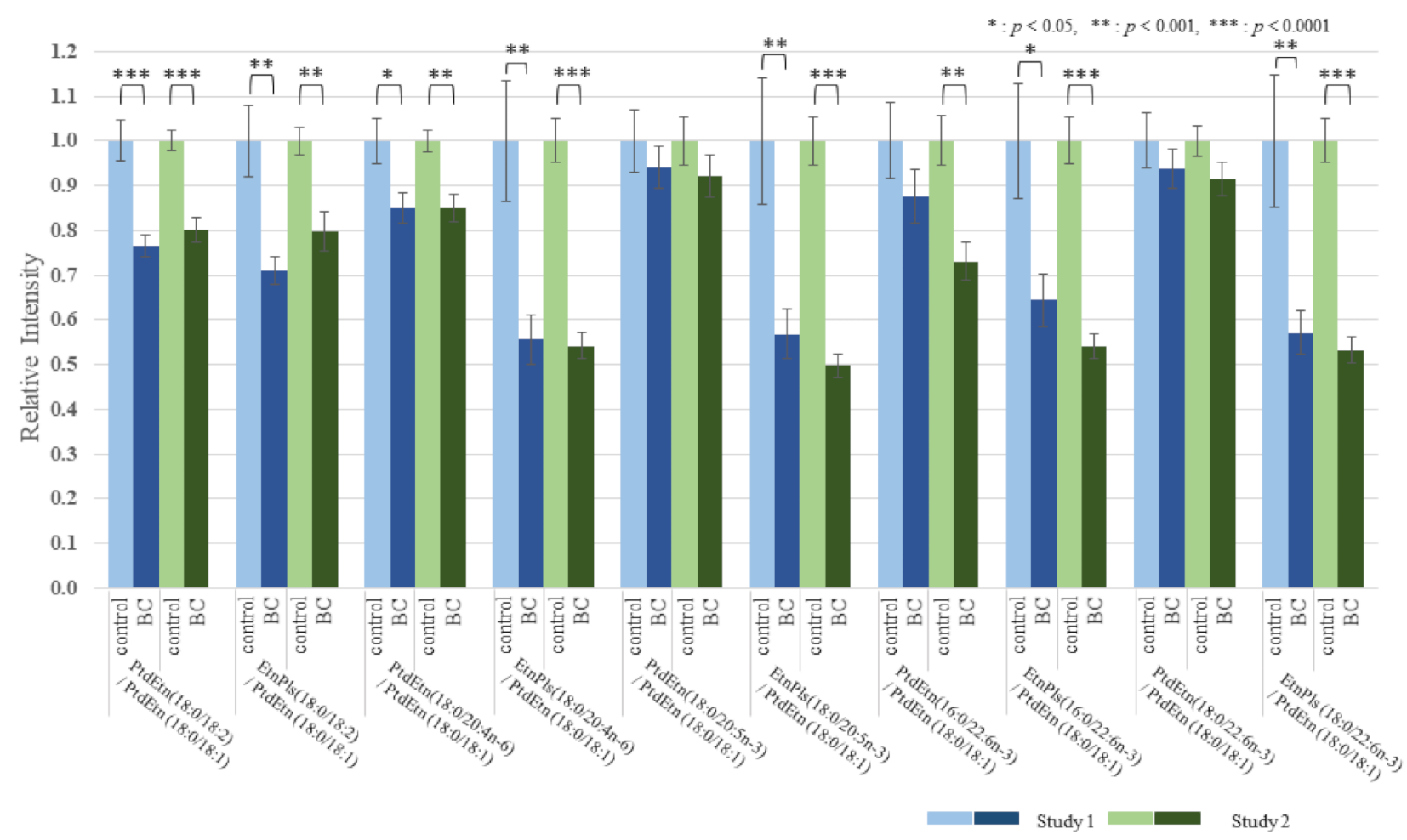

Figure 2. Selected PtdEtn and EtnPls species normalized to PtdEtn 18:0/18:1. (Control means as 1.00 vs. BC means as relative intensity to control). 


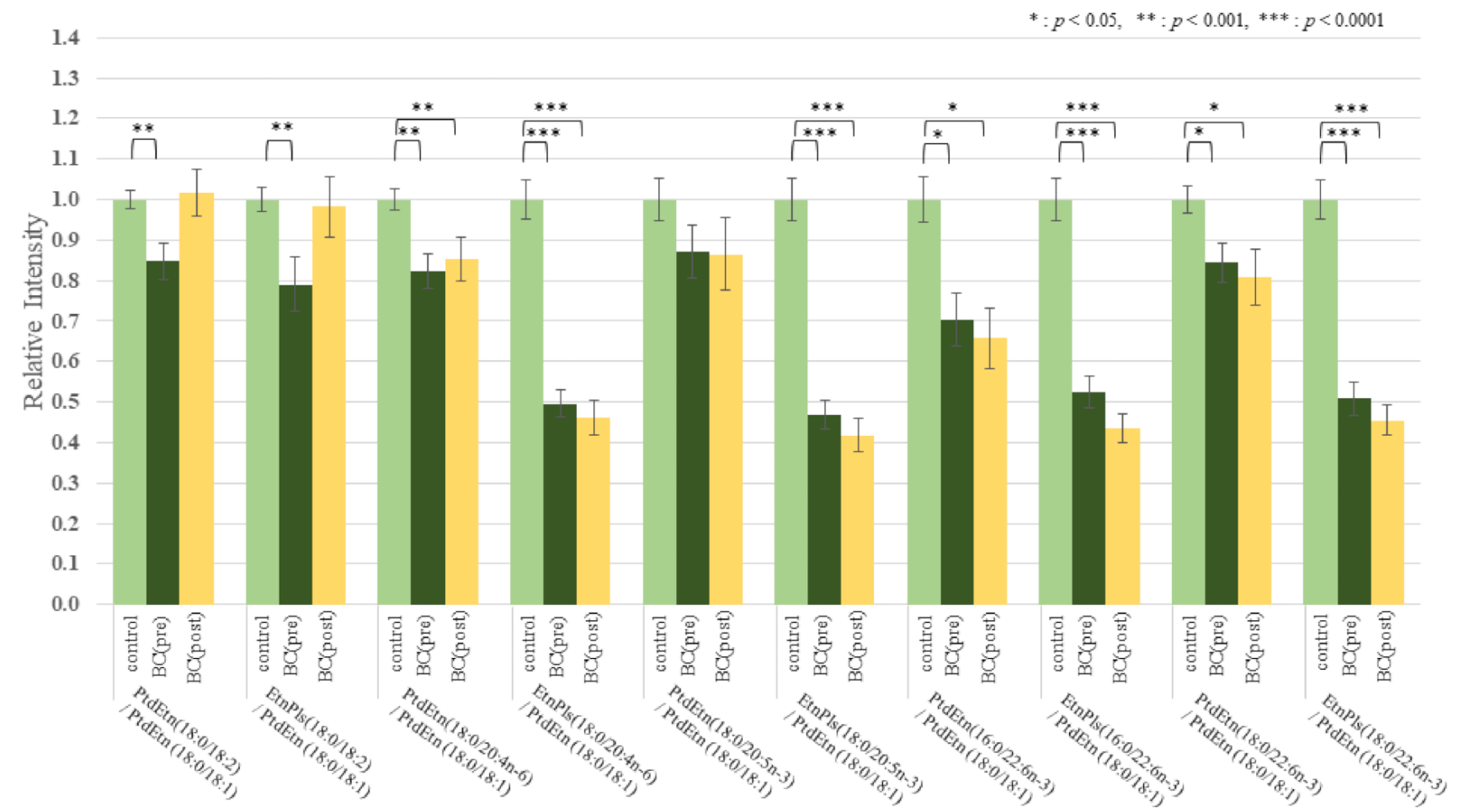

Figure 3. Selected PtdEtn and EtnPls species normalized to PtdEtn 18:0/18:1 in study 2. (Control means as 1.00 vs. pre- or post-surgery BC means as relative intensity to control).
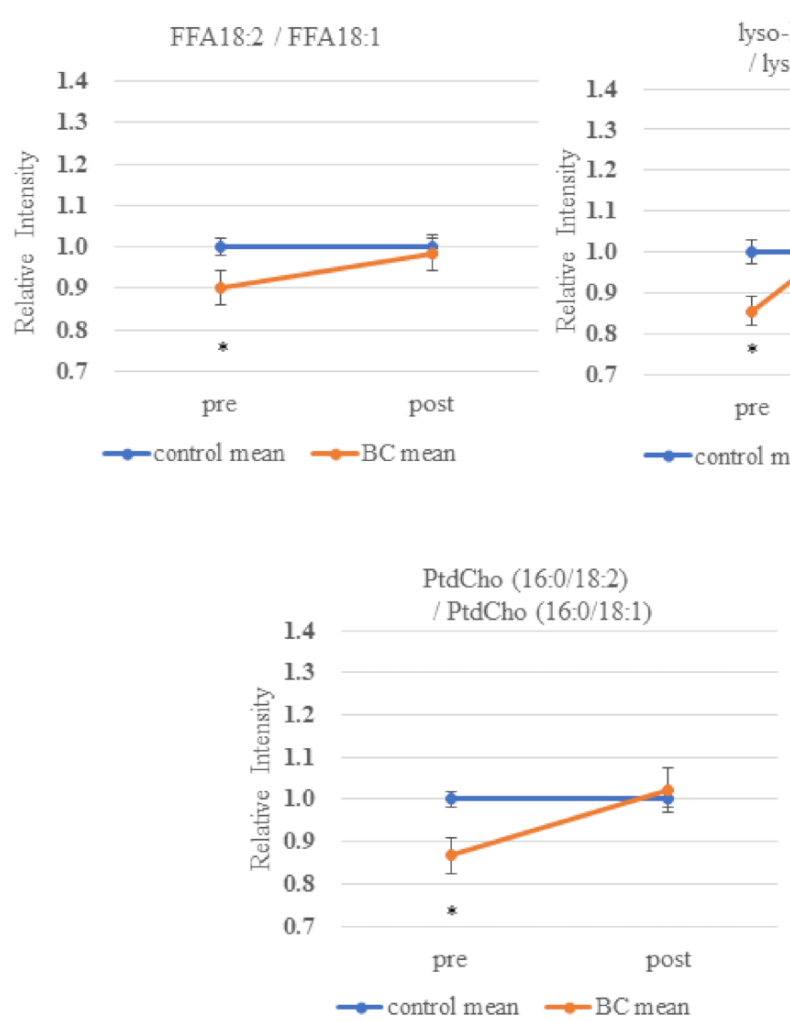

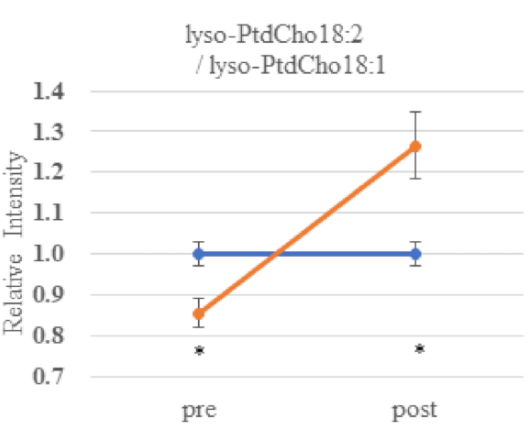

$\rightarrow$ control mean $\longrightarrow \mathrm{BC}$ mean

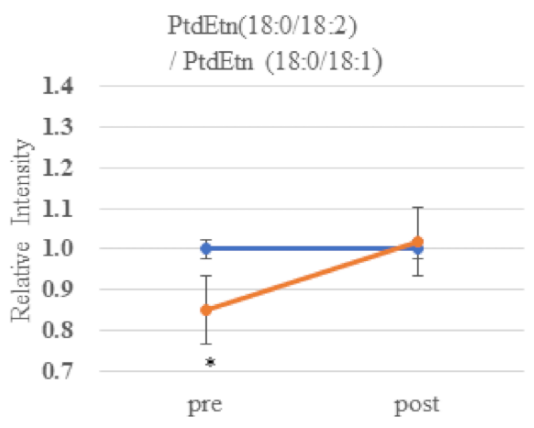

$\rightarrow$ control mean $\longrightarrow$ BC mean

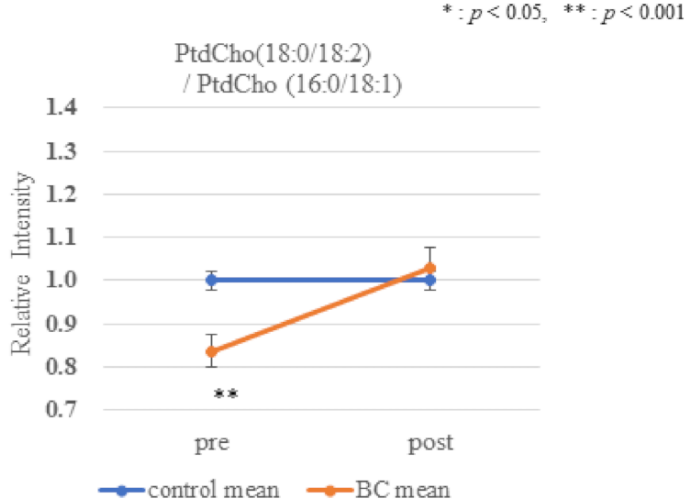

Figure 4. Intensity of the 18:2/18:1 ratios of pre and post-surgery BC relative to the 18:2/18:1 ratios of control in study 2. (Control means as 1.00 and BC means as relative intensity to control). 


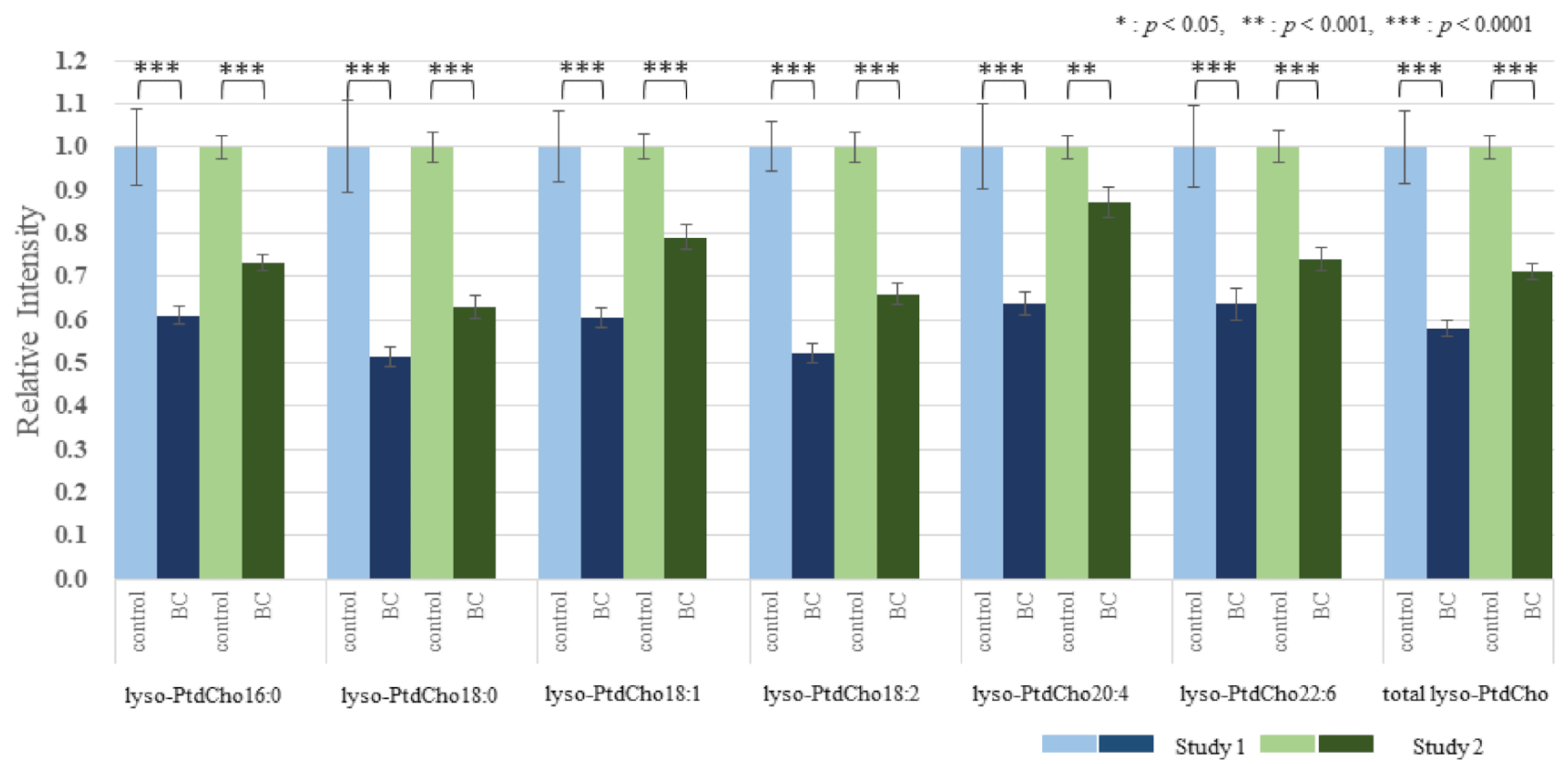

Figure 5. Total species of lyso-PtdCho in pre-surgery BC in both studies. (Control means as 1.00 vs. BC means as relative intensity to control).

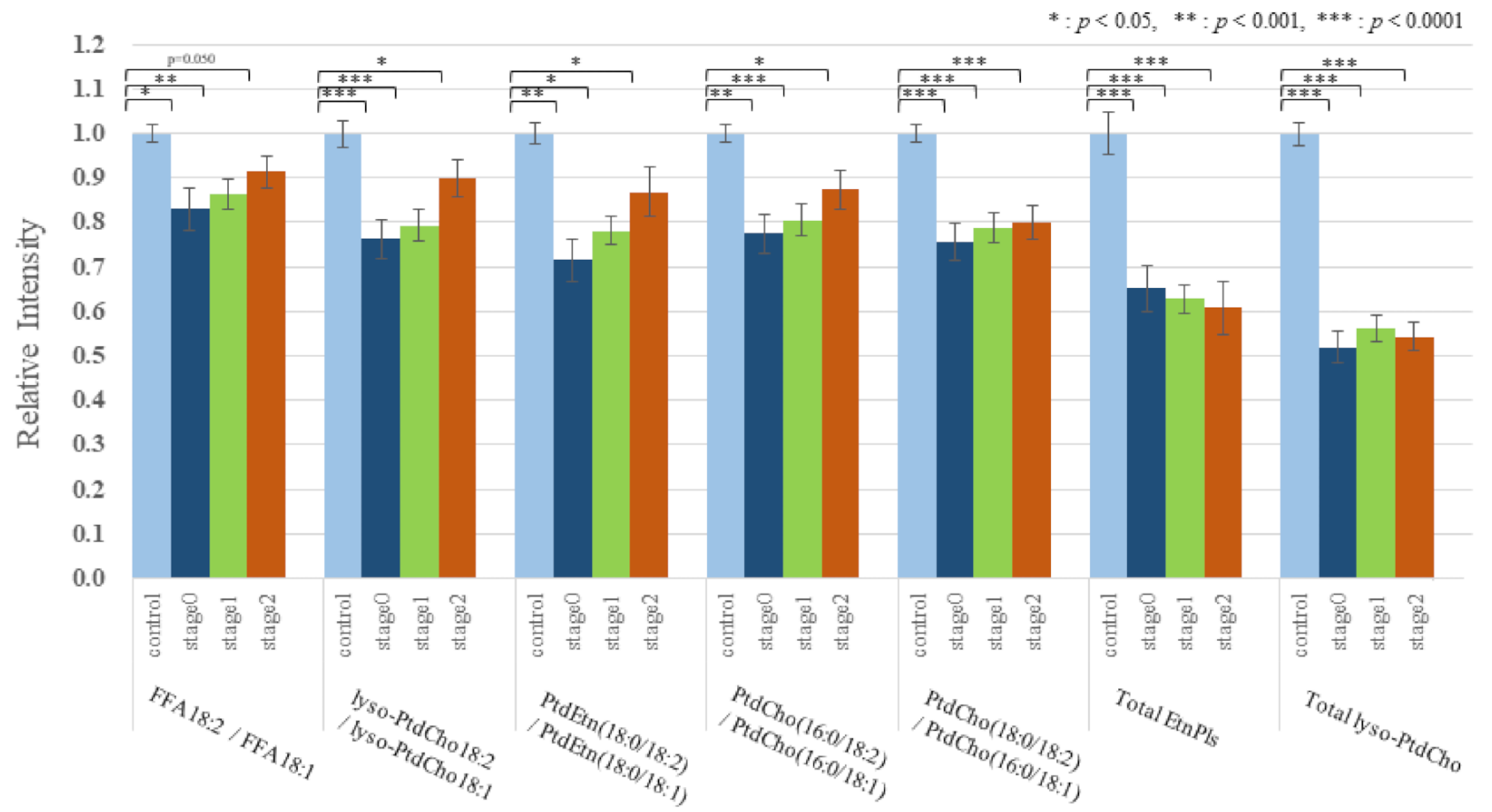

Figure 6. Intensity of the 18:2/18:1 ratios of each stage relative to control in study 2. (Control means as 1.00 and BC means as relative intensity to control).

Despite the consistency of the above group effects, variability existed within individual persons as to which lipid pools were most affected in BC. To determine the accuracy of the observed biochemical changes in diagnosing BC, we generated a multivariate model comprising the 18:2/18:1 ratio, total EtnPls, and total lyso-PtdCho. This approach resulted in excellent sensitivity and specificity as shown by an ROC curve with an AUC of 0.930 in study 2 as the validation phase (Figure 7). 


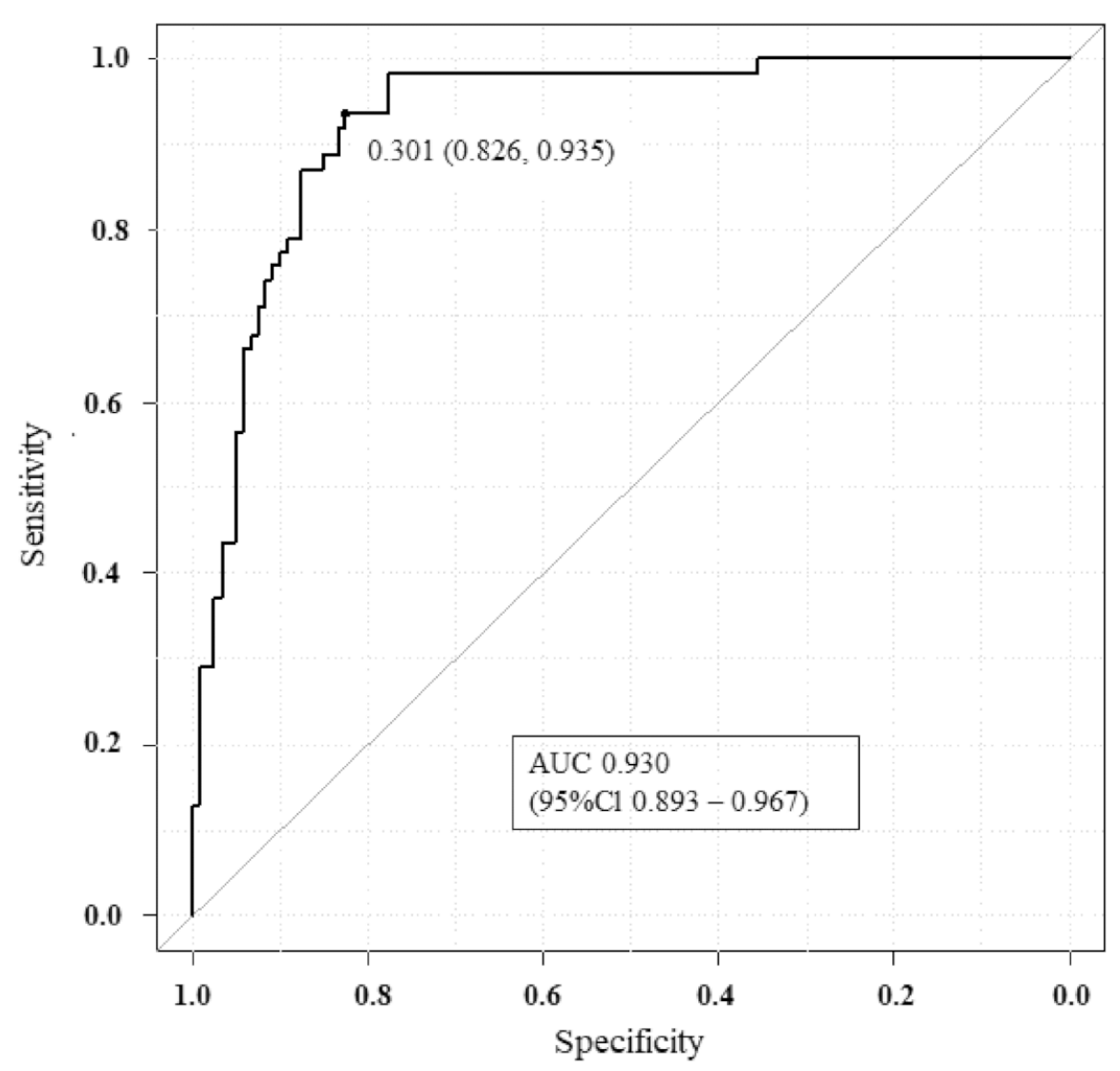

Explanatory variables:

FFA18:2/FFA18:1, lyso-PtdCho18:2/1yso-PtdCho 18:1,

PtdEtn(18:0/18:2)/ PtdEtn(18:0/18:1), PtdCho(16:0/18:2)/PtdCho (16:0/18:1),

PtdCho(18:0/18:2)/PtdCho (16:0/18:1), total EtnPls, total lyso-PtdCho

Figure 7. The ROC curve of control vs. BC in study 2 with AUC $=0.930$ is described by the combination of 18:2/18:1 series, total EtnPls, and total lyso-PtdCho.

\section{Discussion}

In the present study, we used a non-targeted lipid metabolomics discovery platform to identify putative lipid metabolic systems of interest in BC. This approach has been successfully used previously to identify biomarkers that play roles in Alzheimer's disease [12], autism [13], colorectal cancer [14,15], and pancreatic cancer [16,17]. Using this strategy, we identified several lipid metabolomic systems that were potentially altered in persons with BC. To eliminate false interpretations due to potential variations in analytical or clinical collection procedures, the original discovery serum samples were re-extracted and re-analyzed along with serum samples collected from a second case-control cohort recruited at a different time. We, therefore, can report with confidence that persons with $\mathrm{BC}$ have a lower 18:2/18:1 ratio, lower EtnPls levels, and lower lyso-PtdCho levels than persons without BC. Furthermore, of the biochemical systems we confirmed to be altered in persons with $B C$, only the 18:2/18:1 ratios returned to normal following surgical tumor removal. All other observed changes either remained the same or worsened. These observations indicate that two overlapping biochemical dysfunctions occur in BC. One type, exemplified by low EtnPls and lyso-PtdCho levels, predates tumor formation and may represent an increased susceptibility to BC. Another type, exemplified by the low 18:2/18:1 ratios, occurs only during tumor formation and growth.

We hypothesize that the observed selective depletion of 18:2-containing lipid species relative to 18:1-containing lipid species (Figure 1) occurs consequent to an upregulation of 
the polyunsaturated fatty acid elongase, ELOVL5. Fatty acid elongases play an important role in the synthesis of very long-chain fatty acid-containing membrane lipids from shorter chain dietary precursors. 20:4n-6 and 22:6n-3 containing membrane lipids are the main products of these reactions. Mammals have 7 elongases (ELOVL1-7) each of which has different substrate specificities [10]. ELOVL5 elongates $n-3$ and $n-6$ polyunsaturated acids and prefers $\mathrm{C} 18-\mathrm{C} 22$. A previous study has shown that the level of 18:2 was lower in BC tissues and was suggested to accelerate elongation due to ELOVL5. The mRNA expressions of ELOVL1, 5, and 6 were significantly higher in BC tissues than in the corresponding normal breast tissues [11]. Our results agree with these reports. In our study, the 18:2/18:1 ratios within multiple lipid classes were significantly decreased in BC patients prior to surgery. One-month postoperatively, these ratios were elevated compared with the preoperative ratios and were not significantly different from those of the healthy controls. These results indicate that the overactivity of ELOVL5 is limited to the BC tumor and that surgical tumor removal eliminates this effect of the overactivity on serum lipids. The levels of the products of n- 6 and n-3 elongation and desaturation (20:4n-6 and 22:6n-3) were also observed to be decreased in BC (Figure 2). However, unlike the precursor 18:2, the levels of these products were not restored following surgical tumor removal (Figure 3). The lack of effect of tumor removal on systemic (blood) levels of 20:4n-6 and 22:6n-3, further supports our hypothesis that the excess demand of these metabolites was localized to the tumor.

The levels of PlsEtn containing 20:4n-6 and 22:6n-3 in BC subjects are consistently lower relative to the levels of PtdEtn containing 20:4n-6 and 22:6n-3 in BC subjects (Figure 3). EtnPls are manufactured in peroxisomes. Peroxisomal beta-oxidation generates acetylCoA. Within the peroxisome, this acetyl-CoA is used to synthesize the fatty alcohol that becomes the sn-1 vinyl ether of EtnPls. Peroxisomal acetyl-CoA is also the main source of cytosolic acetyl-CoA. Cytosolic acetyl-CoA is an obligate building block used for fatty acid elongation. In addition, peroxisomal beta-oxidation is the final step in the biosynthesis of 22:6n-3. Accordingly, impaired peroxisomal beta-oxidation would result in a simultaneous decrease in the levels of EtnPls, 22:6n-3, and 20:4n-6. A pre-existing state of impaired peroxisomal function as a risk factor for $\mathrm{BC}$ is consistent with these observations.

The physiological importance of EtnPls has been studied extensively [18]. The roles of EtnPls in mammalian physiology can be broadly grouped into two categories: membrane structure and function and antioxidation. EtnPls are essential for maintaining and regulating membrane cholesterol levels [19], membrane fusion [20], and virtually all membrane functions related to fluidity and fusion. Clinically, low serum EtnPls levels are observed in various cancers [21-23] and cardiovascular disease [24,25]. We previously confirmed that serum EtnPls levels were reduced in pancreatic cancer patients [17]. In the present study, we confirmed that total PLE levels were significantly decreased in the sera of BC patients and that they remained significantly lower than those among healthy controls postoperatively. Our study supports the hypothesis that EtnPls downregulation in the sera of $\mathrm{BC}$ patients is not derived from $\mathrm{BC}$ tissues but predates cancer activation.

Considering the mechanistic role of EtnPls in cholesterol regulation [19], particularly high-density lipoprotein (HDL)-mediated cholesterol efflux [26], the long-established role of abnormal intracellular cholesterol regulation in cancer [27-29], and the recent observation that inducing HDL-mediated cholesterol efflux yields an anti-proliferative and pro-apoptotic effect in BC cells [30], it is clear that reduced peroxisomal function, dysregulated intracellular lipid synthesis, and membrane structure defects all contribute to an increased risk of $\mathrm{BC}$.

The histological examination of primary BC tissue [31] indicated that low AMPactivated protein kinase (AMPK) phosphorylation activity is a characteristic of approximately $90 \%$ of $B C$ tumors and that underactive AMPK correlates with overactive acetyl-CoA carboxylase (ACC). The BC susceptibility gene 1 (BRCA1) encodes a protein that interacts and binds to the inactive, AMPK-phosphorylated form of ACC1 [32]. Accordingly, the biological function of BRCA1 is to maintain, extend, and regulate the suppression of ACC1 following its inactivation by AMPK. The BRCA1 protein found in women with BRCA1 
mutations is unable to perform this essential function. Collectively, these data suggest that sporadic and BRCA-mediated BC share a common metabolic path and that the presence of a BRCA mutation dramatically increases the risk of that path being activated.

The cell membrane phospholipid composition affects the protein composition and activity of the cell. We have previously shown that increasing membrane levels of 22:6n-3 EtnPls increases the levels of cholesterol esterifying enzymes [19] and non-amyloidogenic amyloid precursor processing enzymes [33]. Recently, it has been shown that 22:6n-3 supplementation increases the membrane 22:6n-3 levels in rats and that this increase resulted in a decreased incidence of experimentally induced BC [34]. It is of particular interest that the level of the BRCA1 protein was elevated in 22:6n-3-supplemented animals. Treatment of breast and ovarian cancer cells with 22:6n-3 has resulted in consistent positive effects [35-37]. Several studies have now shown that high dietary consumption of omega-3 fatty acids is associated with a decreased risk of BC [38].

Collectively, the results indicate that the low 22:6n-3 EtnPls level observed in BC could increase the susceptibility of cells to the overactivity of ACC by reducing the expression of BRCA proteins and reducing the ability of the cell to counteract this overactivity by reducing cholesterol efflux. Accordingly, n-3 supplementation and exercise are complementary peroxisomal stimulators and have value in BC prevention [39].

In addition to fully intact phospholipids, we also measured lyso-PtdChos. These levels were decreased in postoperative BC patients. The lyso-PtdCho level has been reported to be decreased in several cancers [40]. We also observed low lyso-PtdCho levels in our study of pancreatic cancer [17]. In addition, high baseline levels of lyso-PtdCho are associated with a reduced risk of future incidence of breast, prostate, and colon cancer [41]. Lyso$\mathrm{PtdCho}$ is a metabolic product generated from PtdCho by phospholipase A2 (PLA2) or lecithin-cholesterol acyltransferase (LCAT) [42]. Alternatively, lyso-PtdCho is consumed by the activity of lyso-PtdCho acyltransferase 1 (LPCAT1). Increased turnover of PtdCho and higher consumption of lyso-PtdCho were observed in malignant tumor cells [43]. The overexpression of LPCAT1 has been described in several cancers [44] and the LPCAT1 protein was also significantly upregulated in primary breast carcinoma tissues compared with normal breast tissues [44,45]. LPCAT1 is the most important enzyme in membrane biogenesis in the lipid remodeling pathway referred to as the Lands cycle and was recently isolated [46]. Our results are consistent with these reports.

The main strength of this study includes the discovery and validation study phase, and the changes in the same patients' pre-surgery and post-surgery status. Our study also has some limitations. Post-surgery follow-up is only one month later. It is necessary to demonstrate how EtnPls and lyso-PtdCho levels change by long-term post-surgery follow-up. Next, there were a relatively small number of participants and they were only Japanese, which may limit the generalizability of our findings. Therefore, the involvement of large trials from multiple ethnic groups is needed to better assess breast cancer and the metabolic signatures of lipids.

\section{Materials and Methods}

\subsection{Study Cohorts}

Figure 8 provides a summary of the present study, including the discovery phase in study 1 (identify dysregulated lipid metabolic systems with BC among 2000 unique sample-specific accurate masses) and the validation phase (investigate the selected lipid metabolites in both studies, to confirm the reproducibility in study 1 , and to validate in another population in study 2). Pre-surgery blood samples of BC patients were collected in Kyoto Prefectural University of Medicine between May 2017 and December 2017, including 69 samples assessed in study 1 and between October 2018 and July 2019, including 62 samples assessed in study 2 . We collected 31 samples one month after surgery as postsurgery samples of $62 \mathrm{BC}$ patients in study 2 . The study protocol was approved by the Kyoto Prefectural University of Medicine Medical Ethics Board (approval no. ERB-C-1279 all study subjects). Blood samples of healthy controls were collected during the Japan 
Multi-Institutional Collaborative Cohort (J-MICC) study [47,48]. We randomly selected 48 samples for study 1 and 121 samples for study 2 . The subjects were considered cancerfree based on clinical examination and interview. All samples were processed consistently and stored at $-80^{\circ} \mathrm{C}$ until analysis.

\section{Discovery phase \\ Identify dysregulated lipid metabolic systems with breast cancer}

Study $1 \quad$ Breast Cancer $\quad 69$ samples

Healthy control 48 samples

Mass Spectrometry

Pick up significant lipid metabolites:

18:2/18:1 ratio (FFA, lyso-PtdCho, PtdEtn, PtdCho), EtnPls, lyso-PtdCho

Validation phase

Study 1 (reproducibility measurement)

Blinded and remeasured the above lipid metabolites

Study 2 (another population)

Based on study 1 , blinded validation of lipid metabolites

Study2 Breast Cancer 62 samples

Healthy control 121 samples

\section{Additional follow up investigation}

Investigate the alteration pre- to 1-month post-surgery of these metabolites:

Breast Cancer 31 samples of post-operation

( 31 cases of above $62 \mathrm{BC}$ patients of study 2 )

Figure 8. Flow chart of the study participants.

\subsection{Sample Extraction}

Plasma samples were extracted using a modified version of the protocol described by Goodenowe et al. [12]. Briefly, $10 \mu \mathrm{L}$ of serum was diluted with $50 \mu \mathrm{L}$ of $0.1 \%$ formic acid and subjected to extraction three times with $1.0 \mathrm{~mL}$ of acidified ethyl acetate (98:2 ethyl acetate: $0.1 \%$ formic acid). Then, $100 \mu \mathrm{L}$ of a $95 \%$ methanol/water solution was added to $400 \mu \mathrm{L}$ of the combined ethyl acetate fractions and stored at $-80^{\circ} \mathrm{C}$ prior to analysis.

\subsection{Mass Spectrometry Analysis}

Extracts were directly injected into a Thermo Fisher Scientific LTQ Orbitrap mass spectrometer (Thermo Fisher Scientific, Waltham, MA, USA) in both positive and negative ionization electrospray modes at a flow rate of $200 \mu \mathrm{L} / \mathrm{min}$. Full scan mass spectral data were collected for masses of 150-1200 amu at maximum resolution. A common pooled reference serum sample was prepared before the study and aliquots of this pooled serum were extracted with each batch of study samples and run on the mass spectrometer at the beginning, middle, and end of each run batch to monitor and correct for batch-to-batch 
variance throughout the study. Phospholipid species of interest were identified based upon their $[\mathrm{M}-\mathrm{H}]^{-}$or $[\mathrm{M}]^{+}$or $[\mathrm{M}+\mathrm{H}]^{+}$accurate masses (mass accuracy $<1 \mathrm{ppm}$ ). Only accurate mass species represented by single Gaussian peaks subjected to baseline resolution from any surrounding mass peaks were included in the analyses. Typically, a minimum of 5 scans per peak is needed to obtain a reproducible result. In all mass spectrometers operated under standard operating conditions, the signal is proportional to the analyte concentration over 3-5 orders of magnitude, which makes mass spectrometry a popular analytical tool. The intensity of each species was determined by averaging 20 contiguous scans to ensure a robust measurement process.

\subsection{Data Analyses}

The relationship between the absolute abundances of each species and diagnosis and treatment was performed and resulted in the observation that the phospholipid changes were species-specific with some species not changing and the magnitude of changes in the species that were changing was also species-specific (Tables S1 and S2). To reduce withinperson biological variability, a representative unaffected species (18:1-containing species) was used to generate relative abundance ratios for all of the species studied. This improved the precision ( $p$-value) of the observations. Specifically, of each person, we divided all FFA by FFA18:1, all lyso-PtdCho by lyso-PtdCho 18:1, all PtdEtn species by PtdEtn 18:0/18:1, and all PtdCho by PtdCho 16:0/18:1 (Figures 1 and 4). To further illustrate and compare the selective depletion of 18:2 species and EtnPls in BC, representative pairs of PtdEtn and EtnPls species were normalized to PtdEtn 18:0/18:1 (Figures 2 and 3). We describe BC means as relative intensity when control means were 1.00 to articulate the differences.

\subsection{Statistical Analyses}

Continuous variables are expressed as means, and categorical data are expressed as frequencies and proportions. Inter-group comparisons were performed using Welch's $t$-tests for continuous variables. The diagnostic performance (accuracy) of the models was assessed via receiver operating characteristic (ROC) curve analysis. For comparative purposes, the corresponding areas under the curve (AUC) were calculated and reported. The statistical analyses were performed using R version 3.2.3 (http:/ / www.r-project.org, accessed on 24 July 2021), SPSS software, version 27 (IBM Japan, Tokyo, Japan), and STATA ver.15 (Stata Corp, College Station, TX, USA). All statistical tests were two-tailed, and analysis items with $p<0.05$ were considered statistically significant.

\section{Conclusions}

Low EtnPls and lyso-PtdCho levels are robust predictors of BC risk that persist postoperatively. The overactive fatty acid elongase (as measured by a selective depletion of 18:2 containing phospholipids) was a predictor of active tumor presence and returned to normal post-surgery. These results indicate that it may be possible to screen for both $\mathrm{BC}$ risk and $\mathrm{BC}$ activity using a simple blood test.

Supplementary Materials: The following are available online at https: / www.mdpi.com/article/10 .3390 / cancers13164170/s1, Figure S1: Scatter plots of the p-value (log10) based on t-test comparison between control and BC on the metabolites of FFA, Lyso-PtdCho, PtdEtn, and PtdCho series, Table S1: Lipid metabolites observed to have significantly different levels in BC vs. control, Table S2: The data of individual species which were observed to have significantly different levels in control vs. BC (control means as 1.00 and BC means as relative intensity to control).

Author Contributions: Conceptualization, S.T. and T.T.; methodology, D.B.G. and Y.Y.; formal analysis, S.T., D.B.G. and Y.Y.; investigation and data curation, S.T., M.M., E.O., N.K. and T.K.; writing-original draft preparation, S.T. and D.B.G.; writing—review and editing, S.T., D.B.G. and T.K.; visualization, S.T. and D.B.G.; supervision, K.S. and R.U.; project administration, S.T. and D.B.G.; funding acquisition, R.U. All authors have read and agreed to the published version of the manuscript. 
Funding: This study was supported by Grants-in-Aid for Scientific Research for Priority Areas of Cancer (No. 17015018) and Innovative Areas (No. 221S0001) and by the Japan Society for the Promotion of Science (JSPS) KAKENHI Grant (No. 16H06277 [CoBiA]) from the Japanese Ministry of Education, Culture, Sports, Science and Technology.

Institutional Review Board Statement: The study was conducted according to the guidelines of the Declaration of Helsinki, and approved by the Institutional Ethics Committee of Kyoto Prefectural University of Medicine (protocol code ERB-C-1279 approved 11 June 2018 and ERB-E-36-9 approved 6 June 2019).

Informed Consent Statement: Informed consent was obtained from all subjects involved in the study.

Data Availability Statement: The data presented in this study are available on request from the corresponding author.

Conflicts of Interest: The authors declare that they have no conflict of interest.

\section{References}

1. Cancer Statistics in Japan 16. Available online: http://ganjoho.jp/reg_stat/statistics/brochure/backnumber/2016_jp.html (accessed on 24 July 2021).

2. National Cancer Center Center for Cancer Control and Information Services. Available online: https://ganjoho.jp/reg_stat/ statistics/stat/screening.html (accessed on 24 July 2021).

3. Albuquerque, R.C.; Baltar, V.T.; Marchioni, D.M. Breast cancer and dietary patterns: A systematic review. Nutr. Rev. 2014, 72, 1-17. [CrossRef]

4. Dandamudi, A.; Tommie, J.; Nommsen-Rivers, L.; Couch, S. Dietary Patterns and Breast Cancer Risk: A Systematic Review. Anticancer Res. 2018, 38, 3209-3222. [CrossRef]

5. De Cicco, P.; Catani, M.V.; Gasperi, V.; Sibilano, M.; Quaglietta, M.; Savini, I. Nutrition and Breast Cancer: A Literature Review on Prevention, Treatment and Recurrence. Nutrients 2019, 11, 1514. [CrossRef]

6. Anjom-Shoae, J.; Sadeghi, O.; Larijani, B.; Esmaillzadeh, A. Dietary intake and serum levels of trans fatty acids and risk of breast cancer: A systematic review and dose-response meta-analysis of prospective studies. Clin. Nutr. 2020, 39, 755-764. [CrossRef]

7. Cao, Y.; Hou, L.; Wang, W. Dietary total fat and fatty acids intake, serum fatty acids and risk of breast cancer: A meta-analysis of prospective cohort studies. Int. J. Cancer 2016, 138, 1894-1904. [CrossRef] [PubMed]

8. Nindrea, R.D.; Aryandono, T.; Lazuardi, L.; Dwiprahasto, I. Association of Dietary Intake Ratio of n-3/n-6 Polyunsaturated Fatty Acids with Breast Cancer Risk in Western and Asian Countries: A Meta-Analysis. Asian Pac. J. Cancer Prev. 2019, 20, $1321-1327$. [CrossRef]

9. Zheng, J.S.; Hu, X.J.; Zhao, Y.M.; Yang, J.; Li, D. Intake of fish and marine n-3 polyunsaturated fatty acids and risk of breast cancer: Meta-analysis of data from 21 independent prospective cohort studies. BMJ 2013, 346, f3706. [CrossRef] [PubMed]

10. Kihara, A. Very long-chain fatty acids: Elongation, physiology and related disorders. J. Biochem. 2012, 152, 387-395. [CrossRef] [PubMed]

11. Yamashita, Y.; Nishiumi, S.; Kono, S.; Takao, S.; Azuma, T.; Yoshida, M. Differences in elongation of very long chain fatty acids and fatty acid metabolism between triple-negative and hormone receptor-positive breast cancer. BMC Cancer 2017, 17, 589. [CrossRef] [PubMed]

12. Goodenowe, D.B.; Cook, L.L.; Liu, J.; Lu, Y.; Jayasinghe, D.A.; Ahiahonu, P.W.; Heath, D.; Yamazaki, Y.; Flax, J.; Krenitsky, K.F.; et al. Peripheral ethanolamine plasmalogen deficiency: A logical causative factor in Alzheimer's disease and dementia. J. Lipid Res. 2007, 48, 2485-2498. [CrossRef]

13. Pastural, E.; Ritchie, S.; Lu, Y.; Jin, W.; Kavianpour, A.; Khine Su-Myat, K.; Heath, D.; Wood, P.L.; Fisk, M.; Goodenowe, D.B. Novel plasma phospholipid biomarkers of autism: Mitochondrial dysfunction as a putative causative mechanism. Prostaglandins Leukot. Essent. Fat. Acids 2009, 81, 253-264. [CrossRef] [PubMed]

14. Ritchie, S.A.; Ahiahonu, P.W.; Jayasinghe, D.; Heath, D.; Liu, J.; Lu, Y.; Jin, W.; Kavianpour, A.; Yamazaki, Y.; Khan, A.M.; et al. Reduced levels of hydroxylated, polyunsaturated ultra long-chain fatty acids in the serum of colorectal cancer patients: Implications for early screening and detection. BMC Med. 2010, 8, 13. [CrossRef] [PubMed]

15. Ritchie, S.A.; Tonita, J.; Alvi, R.; Lehotay, D.; Elshoni, H.; Myat, S.; McHattie, J.; Goodenowe, D.B. Low-serum GTA-446 anti-inflammatory fatty acid levels as a new risk factor for colon cancer. Int. J. Cancer 2013, 132, 355-362. [CrossRef]

16. Akita, H.; Ritchie, S.A.; Takemasa, I.; Eguchi, H.; Pastural, E.; Jin, W.; Yamazaki, Y.; Goodenowe, D.B.; Nagano, H.; Monden, M.; et al. Serum Metabolite Profiling for the Detection of Pancreatic Cancer: Results of a Large Independent Validation Study. Pancreas 2016, 45, 1418-1423. [CrossRef]

17. Ritchie, S.A.; Akita, H.; Takemasa, I.; Eguchi, H.; Pastural, E.; Nagano, H.; Monden, M.; Doki, Y.; Mori, M.; Jin, W.; et al. Metabolic system alterations in pancreatic cancer patient serum: Potential for early detection. BMC Cancer 2013, 13, 416. [CrossRef]

18. Braverman, N.E.; Moser, A.B. Functions of plasmalogen lipids in health and disease. Biochim. Biophys. Acta 2012, 1822, 1442-1452. [CrossRef] 
19. Mankidy, R.; Ahiahonu, P.W.; Ma, H.; Jayasinghe, D.; Ritchie, S.A.; Khan, M.A.; Su-Myat, K.K.; Wood, P.L.; Goodenowe, D.B. Membrane plasmalogen composition and cellular cholesterol regulation: A structure activity study. Lipids Health Dis. 2010,9 , 62. [CrossRef] [PubMed]

20. Glaser, P.E.; Gross, R.W. Rapid plasmenylethanolamine-selective fusion of membrane bilayers catalyzed by an isoform of glyceraldehyde-3-phosphate dehydrogenase: Discrimination between glycolytic and fusogenic roles of individual isoforms. Biochemistry 1995, 34, 12193-12203. [CrossRef] [PubMed]

21. Cífková, E.; Holčapek, M.; Lísa, M.; Vrána, D.; Gatěk, J.; Melichar, B. Determination of lipidomic differences between human breast cancer and surrounding normal tissues using HILIC-HPLC/ESI-MS and multivariate data analysis. Anal. Bioanal. Chem. 2015, 407, 991-1002. [CrossRef]

22. Messias, M.C.F.; Mecatti, G.C.; Priolli, D.G.; de Oliveira Carvalho, P. Plasmalogen lipids: Functional mechanism and their involvement in gastrointestinal cancer. Lipids Health Dis. 2018, 17, 41. [CrossRef]

23. Smith, R.E.; Lespi, P.; Di Luca, M.; Bustos, C.; Marra, F.A.; de Alaniz, M.J.; Marra, C.A. A reliable biomarker derived from plasmalogens to evaluate malignancy and metastatic capacity of human cancers. Lipids 2008, 43, 79-89. [CrossRef]

24. Stenvinkel, P.; Diczfalusy, U.; Lindholm, B.; Heimburger, O. Phospholipid plasmalogen, a surrogate marker of oxidative stress, is associated with increased cardiovascular mortality in patients on renal replacement therapy. Nephrol. Dial. Transplant. 2004, 19, 972-976. [CrossRef]

25. Moxon, J.V.; Jones, R.E.; Wong, G.; Weir, J.M.; Mellett, N.A.; Kingwell, B.A.; Meikle, P.J.; Golledge, J. Baseline serum phosphatidylcholine plasmalogen concentrations are inversely associated with incident myocardial infarction in patients with mixed peripheral artery disease presentations. Atherosclerosis 2017, 263, 301-308. [CrossRef]

26. Mandel, H.; Sharf, R.; Berant, M.; Wanders, R.J.; Vreken, P.; Aviram, M. Plasmalogen phospholipids are involved in HDL-mediated cholesterol efflux: Insights from investigations with plasmalogen-deficient cells. Biochem. Biophys. Res. Commun. 1998, 250, 369-373. [CrossRef]

27. Pedersen, P.L. Tumor mitochondria and the bioenergetics of cancer cells. Prog. Exp. Tumor Res. 1978, 22, $190-274$.

28. Coleman, P.S.; Lavietes, B.B. Membrane cholesterol, tumorigenesis, and the biochemical phenotype of neoplasia. CRC Crit. Rev. Biochem. 1981, 11, 341-393. [CrossRef] [PubMed]

29. Parlo, R.A.; Coleman, P.S. Enhanced rate of citrate export from cholesterol-rich hepatoma mitochondria. The truncated Krebs cycle and other metabolic ramifications of mitochondrial membrane cholesterol. J. Biol. Chem. 1984, 259, 9997-10003. [CrossRef]

30. El Roz, A.; Bard, J.M.; Huvelin, J.M.; Nazih, H. LXR agonists and ABCG1-dependent cholesterol efflux in MCF-7 breast cancer cells: Relation to proliferation and apoptosis. Anticancer Res. 2012, 32, 3007-3013.

31. Hadad, S.M.; Baker, L.; Quinlan, P.R.; Robertson, K.E.; Bray, S.E.; Thomson, G.; Kellock, D.; Jordan, L.B.; Purdie, C.A.; Hardie, D.G.; et al. Histological evaluation of AMPK signalling in primary breast cancer. BMC Cancer 2009, 9, 307. [CrossRef] [PubMed]

32. Moreau, K.; Dizin, E.; Ray, H.; Luquain, C.; Lefai, E.; Foufelle, F.; Billaud, M.; Lenoir, G.M.; Venezia, N.D. BRCA1 affects lipid synthesis through its interaction with acetyl-CoA carboxylase. J. Biol. Chem. 2006, 281, 3172-3181. [CrossRef]

33. Wood, P.; Khan, A.; Mankidy, R.; Smith, T.; Goodenowe, D. Plasmalogen Deficit: A New and Testable Hypothesis for the Etiology of Alzheimer's Disease. In Alzheimer's Disease Pathogenesis-Core Concepts, Shifting Paradigms and Therapeutic Targets; IntechOpen: London, UK, 2011. [CrossRef]

34. Jourdan, M.L.; Maheo, K.; Barascu, A.; Goupille, C.; De Latour, M.P.; Bougnoux, P.; Rio, P.G. Increased BRCA1 protein in mammary tumours of rats fed marine omega-3 fatty acids. Oncol. Rep. 2007, 17, 713-719. [PubMed]

35. Corsetto, P.A.; Montorfano, G.; Zava, S.; Jovenitti, I.E.; Cremona, A.; Berra, B.; Rizzo, A.M. Effects of n-3 PUFAs on breast cancer cells through their incorporation in plasma membrane. Lipids Health Dis. 2011, 10, 73. [CrossRef] [PubMed]

36. Kachhap, S.K.; Dange, P.P.; Santani, R.H.; Sawant, S.S.; Ghosh, S.N. Effect of omega-3 fatty acid (docosahexanoic acid) on BRCA1 gene expression and growth in MCF-7 cell line. Cancer Biother. Radiopharm. 2001, 16, 257-263. [CrossRef]

37. West, L.; Yin, Y.; Pierce, S.R.; Fang, Z.; Fan, Y.; Sun, W.; Tucker, K.; Staley, A.; Zhou, C.; Bae-Jump, V. Docosahexaenoic acid (DHA), an omega-3 fatty acid, inhibits tumor growth and metastatic potential of ovarian cancer. Am. J. Cancer Res. 2020, 10, 4450-4463. [PubMed]

38. Liu, J.; Ma, D.W. The role of n-3 polyunsaturated fatty acids in the prevention and treatment of breast cancer. Nutrients 2014, 6 , 5184-5223. [CrossRef]

39. Abdelmagid, S.A.; MacKinnon, J.L.; Janssen, S.M.; Ma, D.W. Role of n-3 Polyunsaturated Fatty Acids and Exercise in Breast Cancer Prevention: Identifying Common Targets. Nutr. Metab. Insights 2016, 9, 71-84. [CrossRef]

40. Taylor, L.A.; Arends, J.; Hodina, A.K.; Unger, C.; Massing, U. Plasma lyso-phosphatidylcholine concentration is decreased in cancer patients with weight loss and activated inflammatory status. Lipids Health Dis. 2007, 6, 17. [CrossRef]

41. Kuhn, T.; Floegel, A.; Sookthai, D.; Johnson, T.; Rolle-Kampczyk, U.; Otto, W.; von Bergen, M.; Boeing, H.; Kaaks, R. Higher plasma levels of lysophosphatidylcholine 18:0 are related to a lower risk of common cancers in a prospective metabolomics study. BMC Med. 2016, 14, 13. [CrossRef]

42. Sekas, G.; Patton, G.M.; Lincoln, E.C.; Robins, S.J. Origin of plasma lysophosphatidylcholine: Evidence for direct hepatic secretion in the rat. J. Lab. Clin. Med. 1985, 105, 190-194.

43. Baburina, I.; Jackowski, S. Cellular responses to excess phospholipid. J. Biol. Chem. 1999, 274, 9400-9408. [CrossRef] 
44. Lebok, P.; von Hassel, A.; Meiners, J.; Hube-Magg, C.; Simon, R.; Höflmayer, D.; Hinsch, A.; Dum, D.; Fraune, C.; Göbel, C.; et al. Up-regulation of lysophosphatidylcholine acyltransferase 1 (LPCAT1) is linked to poor prognosis in breast cancer. Aging 2019, 11, 7796-7804. [CrossRef] [PubMed]

45. Abdelzaher, E.; Mostafa, M.F. Lysophosphatidylcholine acyltransferase 1 (LPCAT1) upregulation in breast carcinoma contributes to tumor progression and predicts early tumor recurrence. Tumour Biol. 2015, 36, 5473-5483. [CrossRef] [PubMed]

46. Kent, C. Regulatory enzymes of phosphatidylcholine biosynthesis: A personal perspective. Biochim. Biophys. Acta 2005, 1733, 53-66. [CrossRef] [PubMed]

47. Hamajima, N. The Japan Multi-Institutional Collaborative Cohort Study (J-MICC Study) to detect gene-environment interactions for cancer. Asian Pac. J. Cancer Prev. 2007, 8, 317-323. [PubMed]

48. Takeuchi, K.; Naito, M.; Kawai, S.; Tsukamoto, M.; Kadomatsu, Y.; Kubo, Y.; Okada, R.; Nagayoshi, M.; Tamura, T.; Hishida, A.; et al. Study profile of the Japan Multi-institutional Collaborative Cohort (J-MICC) Study. J. Epidemiol. 2020, advpub, JE20200147. [CrossRef] [PubMed] 